\title{
RELACIÓN DE LA INCLINACIÓN DE LA EMINENCIA ARTICULAR CON LAS ALTERACIONES MORFOLÓGICAS DE LOS CÓNDILOS TEMPOROMANDIBULARES
}

\author{
RELATION OF THE OF ARTICULAR EMINENCE INCLINATION WITH \\ MORPHOLOGICAL ALTERATIONS OF TEMPOROMANDIBULAR CONDYLARS
}

Eduardo Miguel Calle Velezmoro ${ }^{1 a}$, Pedro Ballona ${ }^{1 b}$, Rafael Morales Vadillo'c

\section{RESUMEN}

Objetivo: Determinar la relación entre la inclinación de la eminencia articular y la presencia de alteraciones morfológicas de los cóndilos temporomandibulares. Materiales y métodos: Se analizaron 91 pacientes (182 articulaciones temporomandibulares) en tomografías volumétricas de haz cónico pertenecientes a pacientes del Servicio de Radiología de la Clínica Especializada en Odontología de la Universidad de San Martin de Porres desde el año 2014 hasta el 2016. Resultados: Al tener un valor $p<0,01$ podemos observar que existe una relación de la inclinación de la eminencia articular con las alteraciones morfológicas de dicha eminencia. Conclusión: Existe relación de las alteraciones morfológicas de la eminencia articular, solo con la inclinación de la vertiente posterior de dicha eminencia.

Palabras Clave: Cóndilo mandibular, articulación temporomandibular. (Fuente: DeCS).

\section{ABSTRACT}

Objective: To determine the relationship between the inclination of the articular eminence and the presence of morphological alterations of the temporomandibular condyles. Materials and methods: We studied 91 patients (182 temporomandibular joints) convex-beam volumetric tomograms belonging to patients of the Radiology Service of the Clinic Specialized in Dentistry of the San Martin de Porres University from 2014 to 2016. Results: When we have a value $p<0.01$ we can see that there is a relation of the inclination of the articular eminence with the morphological alterations of this eminence. Conclusions: Exist morphological alterations of the articular eminence only with the inclination of the posterior slope of this eminence.

Key words: Mandibular Condyle, temporomandibular joint. (Source: MeSH NLM)

Este es un artículo Open Access distribuido bajo la licencia Creative Commons Atribución-NoComercialCompartirlgual 4.0

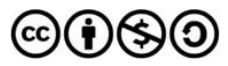




\section{INTRODUCCIÓN}

En la actualidad existen tratamientos en los cuales es necesario determinar la inclinación de la eminencia articular (como son los casos de rehabilitaciones complejas, cirugías ortognáticas, segmentación de rama mandibular o tratamientos de ortodoncia), la cual puede variar dependiendo de la anatomía del paciente. Además, se debe tener en consideración la poca información existente en tomografía volumétrica de haz cónico (TVHC) respecto a la relación entre las alteraciones morfológicas del cóndilo mandibular y la eminencia articular en relación con el ángulo obtenido por la pared posterior de dicha eminencia con el plano horizontal de Frankfurt.

La disfunción temporomandibular generalmente se acompaña de alteraciones en la morfología del cóndilo mandibular o de la eminencia articular, lo que puede llevar a una rotación posterior del disco articular o a una retracción posterior del cóndilo mandibular por la inserción del músculo temporal (1).

La eminencia articular se ha medido utilizando diferentes métodos: radiografías panorámicas, telerradiografías, fotografías a escala, mediciones directas, artrogramas y análisis de la trayectoria protrusiva del cóndilo(2). Estos métodos muestran un grado variable de convexidad de la eminencia articular; sin embargo, se requiere establecer con precisión cuál es su inclinación porque esta superficie también determina el grado de desplazamiento vertical que se observa al momento de la protrusión mandibular(3).

Aun cuando han sido realizados estudios respecto a la medición de estas estructuras (4-16), se debe considerar que la eminencia articular es una estructura tridimensional, por lo que el instrumento de medición ideal sería la TVHC.

Es por ello importante determinar estos valores y su asociación con otras variables, así como las alteraciones morfológicas de los componentes de la articulación temporomandibular, como el cóndilo mandibular y la eminencia articular.

El objetivo de este estudio fue determinar si existe relación entre la inclinación de la eminencia articular y la presencia de alteraciones morfológicas de los cóndilos temporomandibulares

\section{MATERIALES Y METODOS}

La muestra estuvo conformada por 91 pacientes (182 articulaciones temporomandibulares): tomografías volumétricas de haz cónico pertenecientes a pacientes del Servicio de Radiología de la Clínica Especializada en Odontología de la Universidad de San Martin de Porres desde el año 2014 hasta el 2016.

Se accedió al Servicio de Radiología de la Clínica especializada en Odontología de la Universidad de San Martin de Porres para recolectar las TVHC de 91 pacientes (182 articulaciones temporomandibulares) atendidos entre el año 2014 y el 2016, las cuales fueron tomadas por el equipo Pro 3D Mid (PlanMeca ${ }^{\circledR}$, ciudad, Finlandia), que presenta un kilovoltaje de 90 kV y $10 \mathrm{~mA}$ con un tiempo de exposición de 13,5 segundos. La visualización de las imágenes se hizo a través del software Romexis 3.5 (PlanMeca ${ }^{\circledR}$, Helsinki, Finlandia).

Se registraron los datos de los pacientes a través de la ficha de recolección así como el registro de las angulaciones de la vertiente posterior de la eminencia articular, para lo cual se usó como referencia la intersección del plano de Frankfurt, el cual se inicia en el punto cefalométrico Porion (el punto más alto del agujero auditivo externo) y Orbital (el punto inferior más anterior del reborde infraorbitario) (Figura 1).

Posteriormente, se analizó la morfología de la eminencia articular clasificándola en los 4 tipos mencionados por Kurita et al. (2000): en forma de caja, sigmoidea, aplanada y deformada (Figura 2). Luego analizamos la morfología condilar basándonos en los cuatro tipos que son el normal, aplanado, con osteofito y deformado. También se analizaron las relaciones molares de cada paciente (clase I, II, III) para finalmente ver la relación entre estas (Figura 3).

Las tomografías fueron analizadas por 2 examinadores previamente calibrados mediante el Índice de Kappa en una computadora iMac all in one (27" Intel Core i5 8GB 1TB Silver) y se visualizaron en un cuarto semioscuro. 


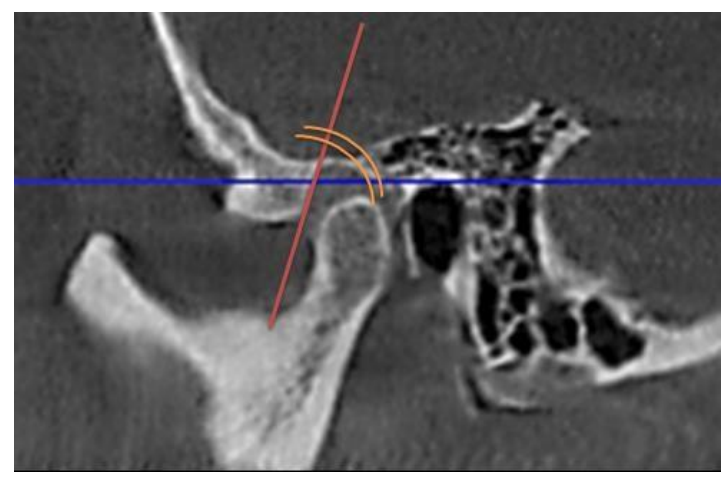

Figura1. Inclinación de la eminencia articular

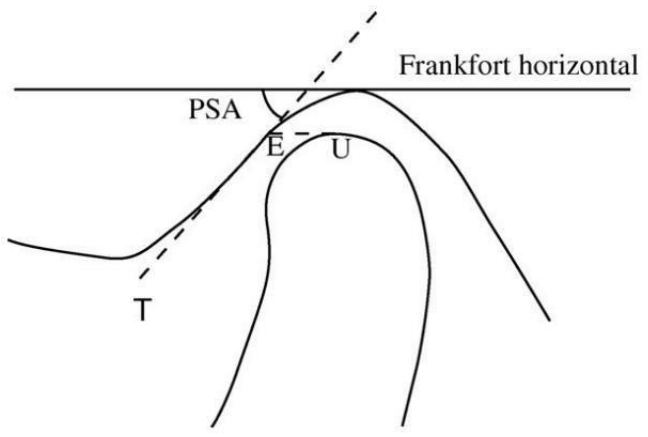

Figura 2. Esquema de la Inclinación de la eminencia articular

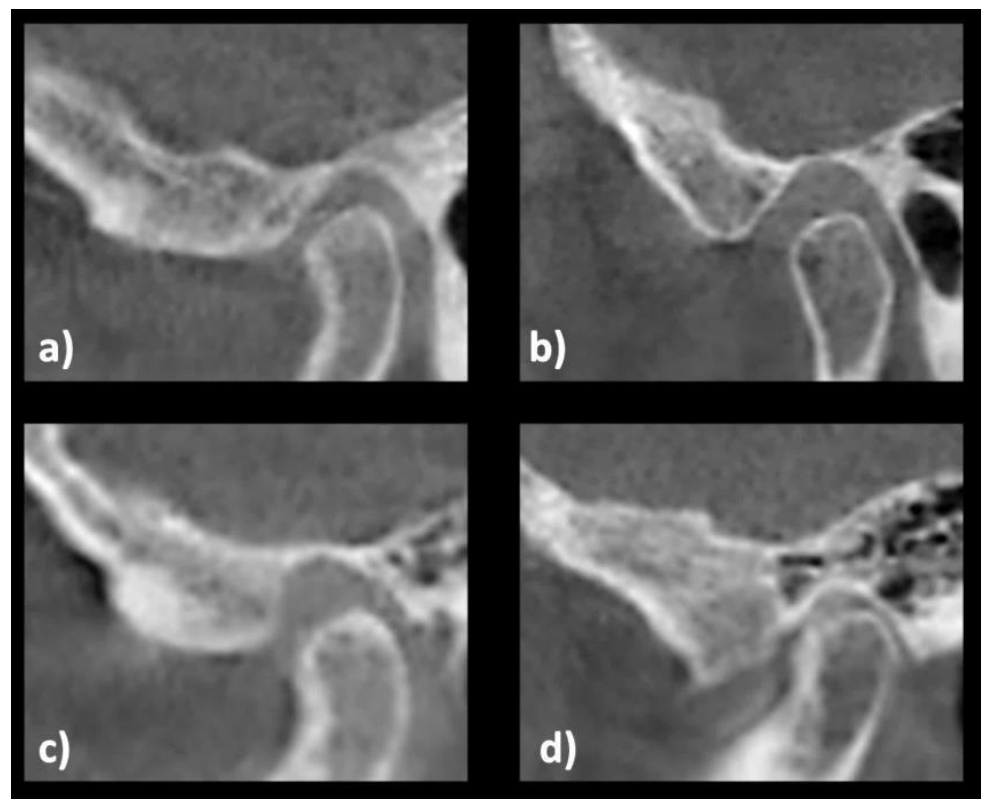

Figura 3. Tipos de alteraciones morfológicas de la eminencia articular: a) Aplanada, b) Sigmoidea, c) Caja y d) Deformada

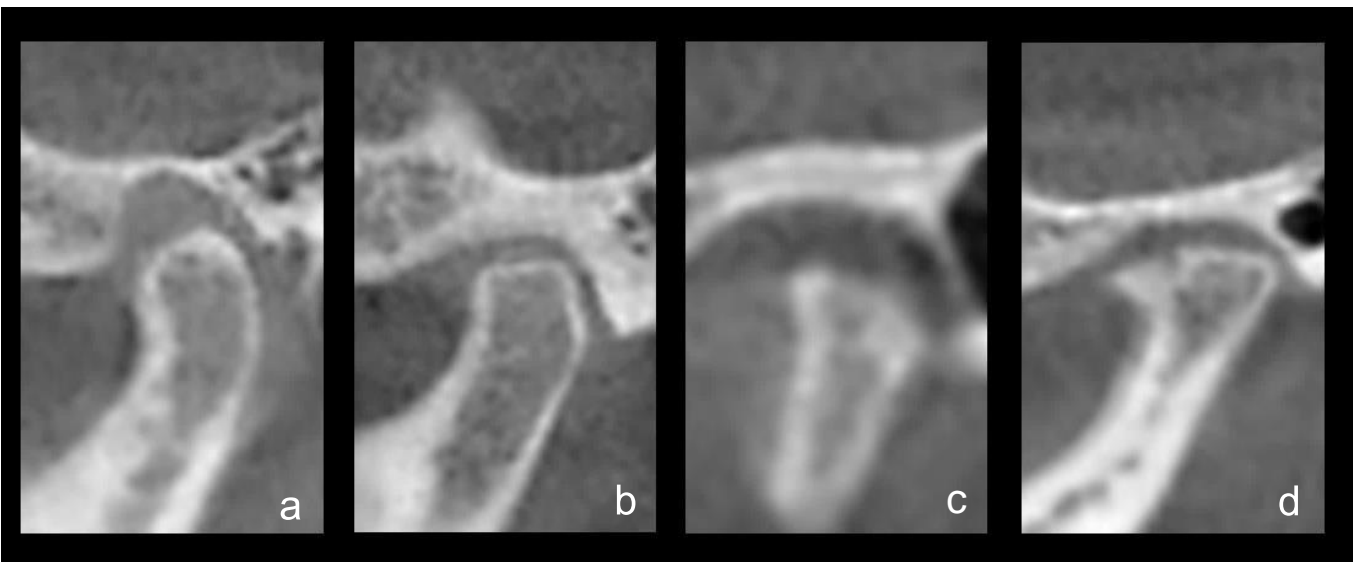

Figura 4. Tipos de alteraciones morfológicas del cóndilo mandibular: a) Normal, b) Aplanado c) Erosión y d) Osteofito 


\section{RESULTADOS}

La inclinación promedio de la eminencia articular en la muestra analizada fue de $50^{\circ}$ con una desviación estándar de $15,9^{\circ}$ (Tabla 1 ). En los resultados de la inclinación de la eminencia articular según las alteraciones morfológicas de ésta (basados en la clasificación de Kurita et al. del año 2000)(11) se encontró que la media de la inclinación de la eminencia según el tipo Caja fue de $65^{\circ}$; en el tipo sigmoideo, de $52^{\circ}$; en el tipo aplanado, de $42^{\circ}$, y en el tipo deformado, de $45^{\circ}$ (Tabla 2 ).
En cuanto a la inclinación de la eminencia articular según las alteraciones morfológicas de los cóndilos mandibulares (basados en la clasificación de Valladares et al. del año 2013) se encontró que la media de la inclinación de la eminencia según el tipo normal fue de $51,8^{\circ}$; el tipo aplanado, de $49^{\circ}$; con erosión, de $41,5^{\circ}$, y con osteolito, $47,9^{\circ}$ (Tabla 3 y figura 3).

Tabla 1. Análisis descriptivo de la inclinación de la eminencia articular

\begin{tabular}{|c|c|c|c|c|c|c|}
\hline \multicolumn{7}{|c|}{ Angulación } \\
\hline $\mathbf{N}$ & & Media & Mediana & $\begin{array}{l}\text { Desviación } \\
\text { estándar }\end{array}$ & Mínimo & Máximo \\
\hline Válido & Perdidos & & & & & \\
\hline 182 & 0 & 50,0704 & 48,4350 & 15,98116 & 16,23 & 106,60 \\
\hline
\end{tabular}

Tabla 2. Análisis descriptivo de la inclinación de la eminencia articular según el tipo de alteración de la eminencia (11).

\begin{tabular}{|c|c|c|}
\hline \multirow{4}{*}{ Caja } & Media & 65,87 \\
\hline & Desviación estándar & 15,14 \\
\hline & Mínimo & 23,20 \\
\hline & Máximo & 90,10 \\
\hline \multirow{4}{*}{ Sigmoideo } & Media & 52,37 \\
\hline & Desviación estándar & 11,94 \\
\hline & Mínimo & 30,34 \\
\hline & Máximo & 80,15 \\
\hline \multirow{4}{*}{ Aplanado } & Media & 42,73 \\
\hline & Desviación estándar & 11,40 \\
\hline & Mínimo & 19,62 \\
\hline & Máximo & 71,50 \\
\hline \multirow{4}{*}{ Deformado } & Media & 45,99 \\
\hline & Desviación estándar & 24,30 \\
\hline & Mínimo & 16,23 \\
\hline & Máximo & 106,60 \\
\hline
\end{tabular}


Tabla 3. Análisis descriptivo de la inclinación de la eminencia articular según el tipo de alteración condilar.

\begin{tabular}{|c|c|c|}
\hline \multirow{4}{*}{ Normal } & Media & 51,86 \\
\hline & Desviación estándar & 15,63 \\
\hline & Mínimo & 20,04 \\
\hline & Máximo & 90,10 \\
\hline \multirow{4}{*}{ Aplanado } & Media & 49,04 \\
\hline & Desviación estándar & 16,46 \\
\hline & Mínimo & 16,23 \\
\hline & Máximo & 106,60 \\
\hline \multirow{4}{*}{ Con erosión } & Media & 41,52 \\
\hline & Desviación estándar & 15,81 \\
\hline & Mínimo & 19,62 \\
\hline & Máximo & 80,39 \\
\hline \multirow{4}{*}{ Con osteofito } & Media & 47,91 \\
\hline & Desviación estándar & 15,99 \\
\hline & Mínimo & 24,94 \\
\hline & Máximo & 71,34 \\
\hline
\end{tabular}

En cuanto al análisis comparativo entre las alteraciones morfológicas de la eminencia, se encontró que el tipo caja es el único que tiene una diferencia significativa en relación con los demás (tipo sigmoideo, aplanado y deformado). Así mismo, al tener un valor $p<0,01$ podemos notar que existe una relación de la inclinación de la eminencia articular con las alteraciones morfológicas de dicha eminencia. (Tablas 4 y 5 ).
Finalmente, en la tablas 6 y 7 apreciamos que no existe diferencia significativa entre las alteraciones morfológicas de los cóndilos mandibulares así como la ausencia de relación entre dichas alteraciones morfológicas con la inclinación de la eminencia articular.

Tabla 4. Comparación de las angulaciones de acuerdo con la morfología de la eminencia articular.

\begin{tabular}{|c|c|}
\hline Prueba de Kruskal-Wallis & . \\
\hline Valor de P & $<0,0001$ \\
\hline ¿Valor de P exacto o aproximado? & $\begin{array}{l}\text { Aproximación } \\
\text { Gaussiana }\end{array}$ \\
\hline Resumen de valor de P & *** \\
\hline ¿Varían las medianas significativamente? $(\mathrm{P}<0,05)$ & Sí \\
\hline Número de grupos & 4 \\
\hline Estadística de Kruskal-Wallis & 55,1 \\
\hline
\end{tabular}

Tabla 5. Prueba pos hoc de comparaciones múltiples entre los tipos de eminencia articular

\begin{tabular}{|l|c|c|c|}
\hline $\begin{array}{l}\text { Prueba de comparación } \\
\text { múltiple de Dunn }\end{array}$ & $\begin{array}{c}\text { Diferencia en la suma } \\
\text { de rango }\end{array}$ & \multicolumn{1}{c}{$\begin{array}{c}\text { Significativo } \\
\mathrm{P}<0,05\end{array}$} & Resumen \\
\hline Caja vs Sigmoideo & 37,1 & Sí & ${ }^{* *}$ \\
\hline Caja vs Aplanado & 72,7 & Sí & ${ }^{* *}$ \\
\hline Caja vs Deformado & 70,6 & Sí & ${ }^{* *}$ \\
\hline Sigmoideo vs Aplanado & 35,7 & Sí & ${ }^{* *}$ \\
\hline Sigmoideo vs Deformado & 33,5 & No & ns \\
\hline Aplanado vs Deformado & $-2,18$ & No & ns \\
\hline
\end{tabular}


Tabla 6. Comparación de las angulaciones de acuerdo con la morfología de los cóndilos mandibulares.

\begin{tabular}{l|c|}
\hline Prueba de Kruskal-Wallis \\
\hline Valor de P & 0,0976 \\
\hline ¿Valor de P exacto o approximado? & Aproximación Gaussiana \\
\hline Resumen de valor de P & ns \\
\hline $\begin{array}{l}\text { ¿Varían las medianas } \\
\text { significativamente?(P }<0,05)\end{array}$ & No \\
\hline Número de grupos & 4 \\
\hline Estadística de Kruskal-Wallis & 6,31 \\
\hline
\end{tabular}

Tabla 7. Prueba pos hoc de comparaciones múltiples entre los tipos de cóndilos mandibulares.

\begin{tabular}{|l|c|c|c|}
\hline $\begin{array}{l}\text { Prueba de comparación } \\
\text { múltiple de Dunn }\end{array}$ & $\begin{array}{c}\text { Diferencia en la } \\
\text { suma de rango }\end{array}$ & $\begin{array}{c}\text { Significativo } \\
\mathrm{P}<0,05\end{array}$ & $\begin{array}{l}\text { Resume } \\
\mathrm{n}\end{array}$ \\
\hline Normal vs Aplanado & 10,7 & No & Ns \\
\hline Normal vs Con erosión & 34,4 & No & Ns \\
\hline Normal vs Con osteofito & 11,2 & No & Ns \\
\hline Aplanado vs Con erosión & 23,7 & No & Ns \\
\hline Aplanado vs Con osteofito & 0,519 & No & Ns \\
\hline Con erosión vs Con osteofito & $-23,2$ & No & Ns \\
\hline
\end{tabular}

\section{DISCUSIÓN}

En el presente estudio se determinó la relación que existe entre la inclinación de la eminencia articular con las alteraciones morfológicas de los cóndilos temporomandibulares (cóndilo mandibular y eminencia articular) ya que en la actualidad existen tratamientos en los cuales es necesario determinar la inclinación de la eminencia articular la cual consta de una inclinación establecida de $30^{\circ}$ y varía dependiendo del paciente.

Además, hay que tener en consideración la poca información existente en tomografía volumétrica de haz cónico respecto a la relación entre las alteraciones morfológicas de los cóndilos temporormandibulares con el ángulo obtenido por la pared posterior de dicha eminencia con el plano horizontal de Frankfurt (Inclinación de la eminencia articular).

Así mismo, por criterios de inclusión y exclusión se analizaron 91 pacientes (182 articulaciones temporomandibulares) de los registros tomográficos del Servicio de Radiología de la Clínica Especializada en Odontología de la USMP.

A lo largo del tiempo, muchos autores han determinado la inclinación de la eminencia articularen diferentes tipos de poblaciones. Sin embargo, estos estudios han sido realizados en imágenes 2D o 3D (siendo el caso de 3D, imágenes obtenidas con tomografía volumétrica de haz cónico, tomografía espiral multicorte y resonancia magnética), como son los casos de Chiang M y cols (Taiwan 2015) $)^{(5)}$, Ren et al. (China 1995)(17), Ichikawa W y et al. (Estados Unidos 1995)(18), los cuales estudiaron la inclinación de la eminencia mediante radiografías panorámicas y/o transcraneales y obtuvieron como resultado $37,7^{\circ}$, $64,4^{\circ}$ y $58,4^{\circ}$, respectivamente. Así mismo, Kranjcic J. et al. (Croacia 2012)(1) estudiaron esta inclinación mediante el análisis fotográfico en cráneos obteniendo como resultado una angulación de $61,56^{\circ}$.

Cabe mencionar que en estos estudios, al ser realizados con imágenes $2 \mathrm{D}$, no se puede realizar una evaluación adecuada de la inclinación de la eminencia articular debido a la superposición de estructuras y en el caso de las investigaciones que utilizaron radiografías panorámicas y/o transcraneales, existe cierto grado de distorsión y magnificación de las estructuras óseas lo cual brinda datos referenciales mas no reales.

Como se mencionó anteriormente, existen estudios realizados en imágenes adquiridas por resonancia magnética como son los de Ozkan $A$ et al. (Turquía 2012)(7), Sülün T. et al. (Alemania 2001) ${ }^{(12)}$, Isberg A. et al. (Estados Unidos 1998)(14) y Galante G (Estados Unidos 1995) (16) los cuales determinaron que la inclinación promedio de la eminencia articular fue de $43,12^{\circ}, 60^{\circ}, 68,7^{\circ}$ y $54,9^{\circ}$, respectivamente. Sin embargo, se sabe que mediante el uso de la resonancia magnética no se puede visualizar en 
detalle las estructuras óseas como se podrían ver en una tomografía volumétrica de haz cónico y/o dependiendo del tipo de ventana de una tomografía espiral multiforme.

Es por ello que llgüy D. et al. (Turquía 2014) ${ }^{6}$, Sumbullu M. et al. (Turquía 2012) ${ }^{8}$ y Estomaguio G. et al. (Japón 2008) ${ }^{(9)}$ realizaron estudios mediante tomografía computarizada, obteniendo como resultado $54,22^{\circ}, 58,46^{\circ}-56,13^{\circ}$ (dependiendo del sexo) y $45,8^{\circ}$, respectivamente, siendo la tomografía volumétrica de haz cónico la más indicada para la evaluación de estas estructuras debido a la poca radiación recibida por parte del paciente, además que nos brinda con mayor detalle la arquitectura ósea. Así mismo, los resultados obtenidos por estas investigaciones fueron los más semejantes a los de la presente investigación, en la cual se determinó que el promedio de la inclinación de la eminencia articularen la población estudiada fue de $50^{\circ}$, lo cual se obtuvo también mediante el uso de la tomografía volumétrica de haz cónico.

En cuanto a las alteraciones morfológicas de los cóndilos temporomandibulares, se optó por usar la clasificación de Kurita et al. del año 2000(13) para las alteraciones de la eminencia articular y la clasificación de Valladares et al. ${ }^{(19)}$ del año 2013 para determinar su relación con la inclinación de la eminencia articular. Sin embargo, la mayoría de autores como Warmling L. y cols (Brasil 2015) ${ }^{(4)}$, Ozkan A, et al. (Turquía 2012)(7), Hirata et al. (Brasil 2007)(10), Tsuruyama K et al. (Japón 2006)(11), y Kurita et al. (Japón 2001)(13) realizaron sus estudios mediante el uso de resonancia magnética e incluso solo hicieron análisis descriptivos en cuanto a la prevalencia de las alteraciones morfológicas de los cóndilos temporomandibulares dejando de lado la relación existente entre estas alteraciones morfológicas y la inclinación de la eminencia.

Es por ello que en el presente estudio, además de ser realizado mediante el uso de la tomografía volumétrica de haz cónico (el cual nos permite una mejor visualización de las estructuras óseas), cuenta con un numero de muestra mayor (91 pacientes) y, al ser el primer estudio de su tipo en ser realizado en nuestro país, se optó por determinar la relación entre las alteraciones morfológicas de los cóndilos temporomandibulares con la inclinación de la eminencia articular basada en la clasificación previamente mencionada. Se encontró que el tipo "caja" es el único que tiene una diferencia significativa en relación con las demás (tipo sigmoideo, aplanado y deformado).

Se halló que existe relación entre las alteraciones morfológicas de la eminencia articular con la inclinación de esta, mientras que las alteraciones morfológicas de los cóndilos mandibulares no se relacionan con esta inclinación. Tampoco se encontró diferencias significativas entre los tipos de alteraciones morfológicas de los cóndilos mandibulares.

Se concluye que solo existe relación de las alteraciones morfológicas de la eminencia articular con la inclinación de la vertiente posterior de dicha eminencia, teniendo esta un promedio de $50^{\circ}$, y que la alteración morfológica del tipo caja es la que tiene una mayor inclinación y una diferencia significativa en relación con las demás.

\section{Contribuciones de autoría:}

ECV diseño el estudio, recolectó, analizó los datos y redactó el artículo. PBCH fue asesor del estudio. RMV brindo asesoría estadística. Todos os autores aprobaron la versión final del artículo.

\section{Fuentes de Financiamiento:}

El estudio fue financiado por los autores.

\section{Conflictos de Interés:}

Los autores manifestaron no tener conflicto de interés en la publicación de este artículo.

\section{REFERENCIAS BIBLIOGRÁFICAS}

1. Kranjcic J, Vojvodic D, Zabarovic D, Vodanovic M, Komar D, Mehulic K. Differences in articular-eminence inclination between medieval and contemporary human populations Achieves of Oral Biology. 2012; 57: 1147 1152.

2. Katsavrias $\mathrm{E}$. Changes in articular eminence inclination during the craniofacial growth period. Angle Orthodontist. 2002; 72 (3): $258-264$.

3. Okeson J, De Leeuw R. Differential diagnosis of temporomandibular disorders and other orofacial pain disorders. Dental Clinic North America. 2011; 55: 105 20.

4. Warmling L, Pereyra M, Panelli K, Oliveira J. Evaluation Retrodiskal tissue associated with articular eminence morphology, effusion status and disk displacement in symptomatic patients. J. int. dent. med. res. 2015; 8 (1): $1-6$.

5. Chiang M, Li T, Yeh H, Su C, Chiu K, Chung $M$ et al Evaluation of missing-tooth effect on articular eminence inclination of temporomandibular joint J Dent Sci. 2015; 20: $1-5$.

6. Ilguy D, Ilguy M, Fisekcioglu E, Dolekoglu S, Ersan N. Articular Eminence Inclination, height and condyle morphology on Cone Beam Computed Tomography ScientificWorldJournal. 2014; 1 - 6 .

7. Ozkan A, Ayberk H, Sencimen M, Senel B Evaluation of articular eminence morphology and inclination in TMJ internal derangement patient with MRI Int. J. Morphol. 2012; 30 (2): 740 -744.

8. Sumbullu M, Caglayan $F$, Akgul $H$, Yilmaz A. Radiological examination of the articular eminence morphology using cone beam CT Dentomaxillofac Rad. 2012; 41: $234-240$.

9. Estomaguio G, Yamada K, Saito I Unilateral condylar bone change, inclination of the posterior slope of the articular eminence and craniofacial morphology Ortodontic Wares. 2008; $113-119$. 
10. Hirata E, Guimaraes A, Oliveira J, Moreira C, Terra E, Paraiso $M$ Evaluation of TMJ articular eminence morphology and disc patterns in patients with disc displacement in MRI Braz Oral Res. 2007; 21 (3): 265 $-271$.

11. Tsuruyama $K$, Nishimura $H$, Motoda $E$, Maeda $T$ Morphological changes of the articular eminence and the anterior disc displacement in youngsters $\mathrm{J}$ Dent Child. 2006; 16 (2): 145 - 153.

12. Sulun T, Emgil T, Pho Duc J, Rammeksberg $P$, Jager L, Gernet W Morphology of the mandibular fossa and inclination of the articular eminence in patients with internal derangement and in symptom - free volunteers Oral Surg Oral Med Oral Pathol. 2001; 92 (1): 98 -108.

13. Kurita $H$, Ohtsuka $A$, Kobayashi $H$, Kurashina $K$ Is the morphology of the articular eminence of the temporomandibular joint a predisposing factor for disc displacement? Dentomaxillofac Rad. 2000; 29: 159 162.

14. Isberg A, Westesson $P$ Steepness of articular eminence and movement of the condyle and disk in asymptomatic temporomandibular joints. Oral Surg Oral Med Oral Pathol. 1998; 86: $152-157$.
15. Sato S, Kawamura H, Motegi K, Takahashi K Morphology of the mandibular fossa and the articular eminence in temporomandibular joints with anterior disk displacement Int J Oral Maxillofac Surg. 1996; 25: 236 $-238$.

16. Galante G, Paesani D, Tallents R, Hatala M, Katzberg $\mathrm{R}$, Murphy $\mathrm{W}$. Angle of the articular eminence in patients with temporomandibular joint dysfuction and asymptomatic volunteers Oral Surg Oral Med Oral Pathol. 1995; 80: 242 - 249.

17. Ren $Y$, Isberg A, Westesson $P$, Steepness of the articular eminence in the temporomandibular joint Oral Surg Oral Med Oral Pathol. 1995; 80: 258 - 266.

18. Ichikawa W, Laskin D, Rosenberg H Ranscraneal radiographic and tomographic analysis of the lateral and midpoint inclined planes of the articular eminence Oral Surg Oral Med Oral Pathol. 1990; 70: 516 - 522.

19. Valladares J, Cevidanes L, Rocha W, Almeida G, Batista J, Rino J. TMJ response to mandibular advancement surgery: an overview of risk factors. Journal of Applied Oral Science. 2014; 22 (1): 2 - 14. 